\title{
Reciprocal Comparisons, Inversion, and Hyperbolic Geometry From Psychology to Geometry
}

\author{
Thomas L. Saaty \\ University of Pittsburgh
}

\begin{abstract}
It has long been known for example from the works of Henri Poincare that inversion of points with respect to the unit circle can be used to interpret hyperbolic geometry. In this paper we note that inversion is simply the reciprocal relation in paired comparisons. We then argue that to compare the intensities of sensations which we all do consciously and unconsciously we need a metric. A metric is associated with a geometry. The process of reciprocal comparisons is the minds tool for creating a metric across the senses. We refer to Descartes observation that synthesis of data from the several senses leads to a unified Euclidean metric and argue for replacing it with the hyperbolic metric.
\end{abstract}

Keywords: Inversion, reciprocal comparisons, metric, hyperbolic geometry, synthesis, sensation

\section{Introduction}

Inversion is a process in which a point $\mathrm{A}$ inside the unit sphere is associated with a point $A^{\prime}$ outside which falls on the ray from the center $O$ of the sphere through $A$, such that the product of the distances $O A$ and $O A^{\prime}$ from $O$ is equal to one. As one point moves on the ray towards the boundary so does the other, and as $\mathrm{A}$ moves towards $\mathrm{O}, A^{\prime}$ moves towards infinity. Inversion is a process for representing hyperbolic geometry in the Euclidean space. The purpose of this paper is 1) to show that inversion arises from reciprocal paired comparisons; 2) that both discrete and continuous reciprocal paired comparisons give rise to a ratio scale metric; and 3) that paired comparisons, a common process associated with sensation and thought yield uniformly across the senses an identical (ratio scale) metric associated with hyperbolic geometry. Descartes observed long ago that geometric order is common to and transcends vision, hearing and feeling. Overwhelming evidence from experimental psychology indicates that blind people and also people who are both deaf and blind can order the world as sighted people do but with greater effort. Because the ideas of this paper relate the mind to the real world, our approach is directed towards highlighting evidence regarding the uniformity of our sensations, as they map into a single hyperbolic structure arising from our instinctive inclination to make comparisons. It is known that both space-time physics and visual sensation give rise to a hyperbolic metric [15].

People have an ability to make comparisons to estimate relative magnitudes even when they are unschooled in numbers and arithmetic. In fact, deriving measurements from comparisons precedes measuring on uniform scales with a conveniently chosen unit. Henri Lebesgue [13] observed:

It would seem that the principle of economy would always require that we evaluate ratios directly and not as ratios of measurements. However, in practice, all lengths are measured in meters, all angles in degrees, etc.; that is we employ auxiliary units and, as it seems, with only the disadvantage of having two measurements to make instead of one. Sometimes, this is because of experimental difficulties or impossibilities that prevent the direct comparison of 
lengths or angles. But there is also another reason.

In geometrical problems, one needs to compare two lengths, for example, and only those two. It is quite different in practice when one encounters a hundred lengths and may expect to have to compare these lengths two at a time in all possible manners. Thus it is desirable and economical procedure to measure each new length. One single measurement for each length, made as precisely as possible, gives the ratio of the length in question to each other length. This explains the fact that, in practice, comparisons are never, or almost never, made directly but through comparisons with a standard scale.

But we do not always have the needed standard scales and must learn how to do comparisons particularly when one does not have a hundred, but only a few things to measure.

Our purpose here is to show how relative comparisons work and relate them to our perception of geometry. Figure 1 is an attempt to represent how the brain processes information to create "understanding." It shows that creating knowledge is a complex brain process that is characteristic of our own type of thinking. Other living forms, particularly invertebrates and plants have their own way of storing their kind of information, which is likely to be different than ours.

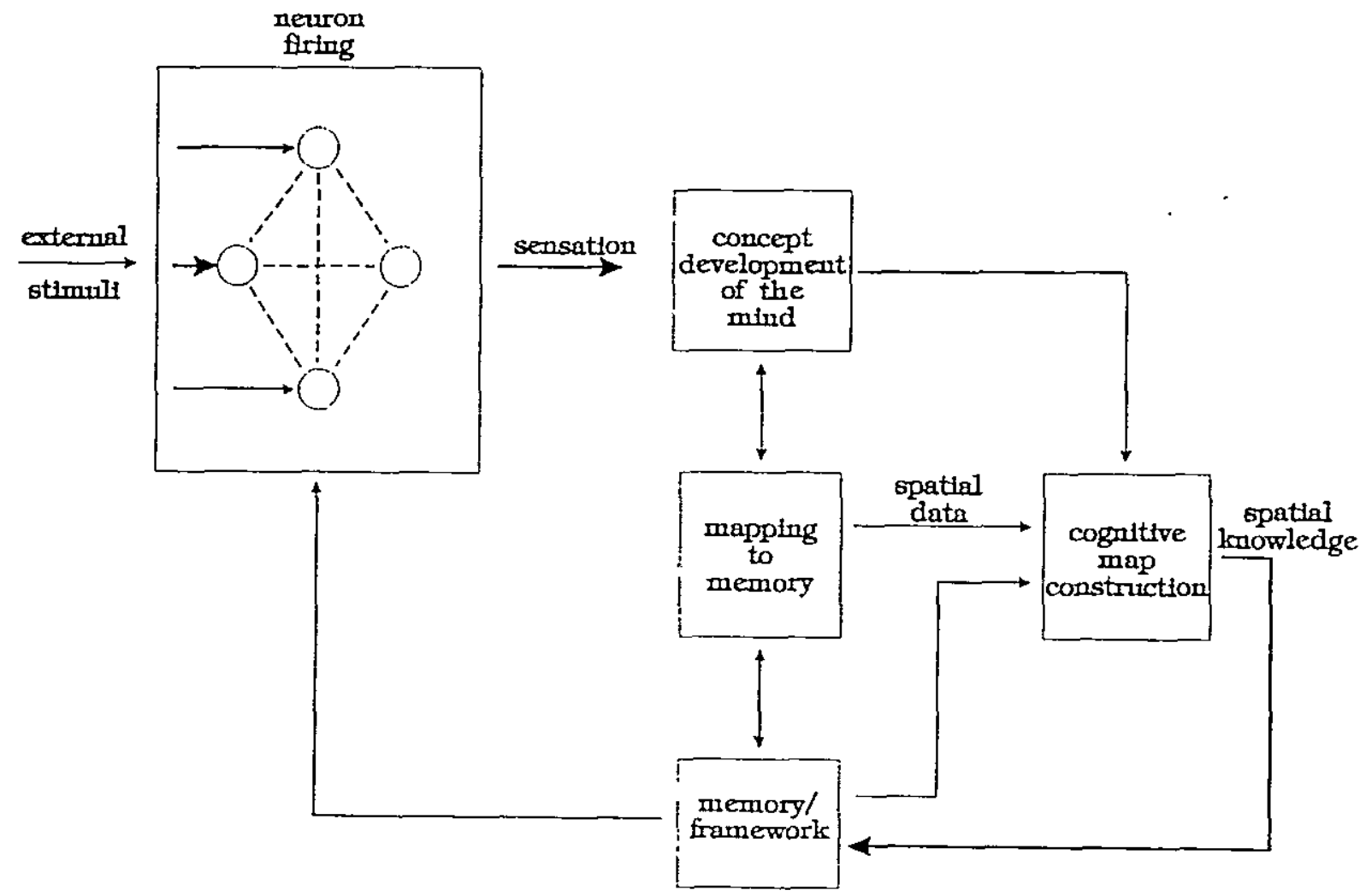

Figure 1

The need for close coordination between the magnitudes of stimuli and those of responses can perhaps be better understood if we are able to identify the functions of the central nervous system provided for us by the French molecular bịologist and noble laureate Jacques Monod [18]. 
- Coordination of neuromotor activity with sense data;

- Providing (innate) preprogrammed responses to particular stimuli;

- To search for, analyze, screen, abstract and synthesize information about the environment into factors suited to the chemical and biological performance of the organism;

- To judge the priority of events in terms of their relevance to performance by grouping them in hierarchic levels of degrees of influence and using them to expand the innate programs with their knowledge;

- To project (in higher vertebrates) and extend the process of judgment and priority to hypothetical areas to create stimuli and envision the role (courses of action) of the organism in diverse environments.

In the remainder of the paper we discuss comparisons and their importance in biology, and in particular reciprocal comparisons and show how they correspond to inversion. We formalize the process of making discrete reciprocal comparisons from observation and judgment and show how to derive a ratio scale from them, illustrating it with an example from optics. We then give a generalization of comparisons to the continuous case of neural firing and show how these in turn give rise to a ratio scale response function. In section 7 we are concerned with how the idea of a metric develops naturally from the totality of impressions one individual gathers and show in the following section the congruence of structures derived through different senses. In section 9 we give a few of the known highlights about inversion and hyperbolic geometry.

\section{On The Making Of Comparisons in Measurement}

Nature exhibits magnitudes in the form of variations in the intensity of properties. We invent scales to measure these variations. Our scales tend to be based on equal multiples and fractions of an arbitrarily chosen unit. The numeral outcome is a special kind of information that needs to be interpreted in some measured and proportional way in terms of our values to determine what exactly it means. The assessment of measurement from scales in terms of our value system itself requires a subjective scale to give it precise content. This type of measurement we do consciously with thought. But much more unconsciously, our bodies, for example, secrete chemicals in precise amounts to respond to physical needs and also to control the expenditure of physical and nervous energy. 'Survival and chemical balance necessitate appropriate and proportional response. We need magnitudes to respond proportionately to stimuli; hence the idea of ratio scales, invariant under a similarity transformation, that is under multiplication by a positive constant. Ratio scales represent proportionality and are essential for muscular control and for most bodily actions and reactions. Ratio scales are needed to perceive forms endowing them with symmetry, balance, beauty and other attributes that make it possible to distinguish among them. Physics relies on ratio scales to describe natural law.

We use comparisons to create ratio magnitudes we spoke of earlier. Comparisons are basic in the functioning of the brain. "Our brain is constantly comparing the differences between the angle from each eye to the sighted object, using feedback from the eye muscles. This is what allows a baseball pitcher to put a ball over the plate with such accuracy" [31]. "It would therefore be difficult to imagine that there is no simple and obvious relationship between the physical characteristics of the object being viewed and the color that the brain assigns to it. Indeed there is. It lies in a comparison of the wavelength composition of the light reflected from a surface and the wavelength composition of the light reflected from surrounding surfaces ... 
color therefore is a comparison of comparisons [32]."

We are concerned with the characterization of the spaces of response for all the senses. Central to this concern is that each of the senses involves intensities of stimuli received and responses to these stimuli. It is essential that with each sense there is associated a metric that enables making distinctions between magnitudes of sensations. Because the brain itself must process and synthesize simultaneously a multiplicity of information from each of the senses and from all the senses together, it is reasonable to assume that the metric of response is the same for all the senses and coincides with the metric used by the brain to compare stimuli from different senses to determine its response. The existence of a metric is characteristic of a space and a geometry. Thus response to sensation has an underlying mathematical structure, i.e. a geometry. It is easy to see why Descartes believed that all these spaces are the same [4]. That was a brilliant observation, except it is not Euclidean but hyperbolic geometry as we show in this paper.

\section{Reciprocal Comparisons}

Magnitude comparison between two objects, on a common property, is established by using the smaller (lesser) object as a unit of measurement and estimating the larger (greater) one as a multiple of that unit. The smaller object then has the reciprocal value of the larger one. It is essential that the smaller or lesser object be first used to estimate the magnitude of the larger object in order to determine the reciprocal value for the smaller one. If the larger object is to serve as the unit, it must be decomposed by using the smaller object as the unit. This is the abstract principle underlying all reciprocal comparisons.

The reciprocal relation between the two objects has the form $y=1 / x$ from which we have the symmetric relation $x y=1$ known as inversion. It assumes that the magnitudes $x$ and $y$ can be established with regard to a third magnitude, which has an arbitrary unit value. This idea plays a central role in Poincare's model of noneuclidean geometry. We note that when $x$ is allowed to vary continuously over the real numbers, $y=1 / x$ gives rise to a hyperbola in the plane; more generally it specifies a ratio in a potential field between two points $\mathrm{A}$ and $\mathrm{B}$ so that $A$ has a potential $1 / x$ from $B$ and $B$ a potential $1 / y$ from $A$.

Reciprocal comparisons are of two kinds: discrete and continuous. As we shall see later, both types of comparisons give rise to numbers that belong to a ratio scale. We use discrete comparisons to determine relative magnitudes arising from the effect of a natural law. For example, we make discrete comparisons to compare the relative brightness of objects. We use continuous comparisons to grasp relations in a form such as the shapes of objects or patterns of sound. We can make continuous comparisons each instant of time when we use the eye. Repetition over time gives it completeness and duration. Unlike the eye, the ear deals with one stimulus at each instant of time or perhaps with a small set of stimuli. The eye uses local patterns as generators interwoven or connected to estimate a. global pattern.

Comparisons require a certain kind of information. When actual numbers are available, they may be used to estimate relative measurement. When there are no scales, one must use judgment to make the estimate. Judgment relies on the ability of the brain to respond accurately to a stimulus of varying intensities. Judgment precedes the existence of a scale. For accuracy, judgment must be confined to a narrow range of discrimination among intensities. For wider ranges one needs to cluster homogeneous elements together and include a common element to act as a pivot from one cluster to the next. A mathematical theory known as the Analytic Hierarchy Process with wide ranging applications which involves the use of both discrete and 
continuous comparisons revolves around deriving ratio scales from reciprocal comparisons. In the next sections we describe this process in greater detail.

\section{The Construction of Ratio Metrics From Comparisons}

The Analytic Hierarchy Process (AHP) is a method of breaking down a complex situation into its component parts, arranging these parts, or variables, into a hierarchic order, assigning numerical values to subjective judgments on the relative importance of each variable, and synthesizing the judgments to determine the overall priorities of the variables. The AHP also provides an effective structure for group decision making by imposing a discipline on the group's thought processes. The necessity of assigning a numerical value to each variable of the problem helps decision makers to maintain cohesive thought patterns and to reach a conclusion. This structure lends itself to those forecasting problems which, require expert opinions regarding the levels of influence of quantitative and/or qualitative factors. The AHP has been applied successfully to a variety of problems: planning [23], prioritization [25], resource allocation, conflict resolution [24], decision making, and forecasting or prediction [26]. The process has three major components:

1. The problem is decomposed into factors or elements. Elements are grouped on different levels. Each element is, in turn, decomposed into another set of elements. The process forming a chain or hierarchy continues to the lowest level of the hierarchy. The hierarchy does not need to be complete; that is, an element in a given level does not have to function as criterion for all the elements in the level below. Thus a given hierarchy can be divided into subhierarchies having only a common topmost element.

2. The degree of relative importance of the elements at a particular level in terms of each parent element in a preceding level is measured by a procedure of paired comparisons carried out by the decision maker(s) and resulting in a matrix of paired comparisons.

3. To compute the priorities of the elements in each matrix of paired comparisons; we solve the principal eigenvalue problem of each matrix. The resulting vector of "local" priorities is then weighted by the weight of the higher-level element used as the criterion in making the pairwise comparisons for the matrix in question. This yields the global priorities of the elements. The global priorities of an element in a given level are added to yield its composite priority. The procedure is repeated by moving downward along the hierarchy, computing the global weight of each element at every level, and using it to determine composite weights of elements in succeeding levels.

A brief mathematical description of the AHP will be given in the following paragraphs. For a detailed description of the method, see [25].

The AHP has four axioms, 1) reciprocal, 2) homogeneous, 3) hierarchic or feedback dependence and 4) expectations about validity of the model and about the derived rank order.

Assume that one is given $n$ stones, $A_{l}, \ldots, A_{n}$, with known weights $w_{l}, \ldots, w_{n}$, respectively, and suppose that a matrix of pairwise ratios is formed whose rows give the ratios of the weights of each stone with respect to all others. Thus one has the equation: 


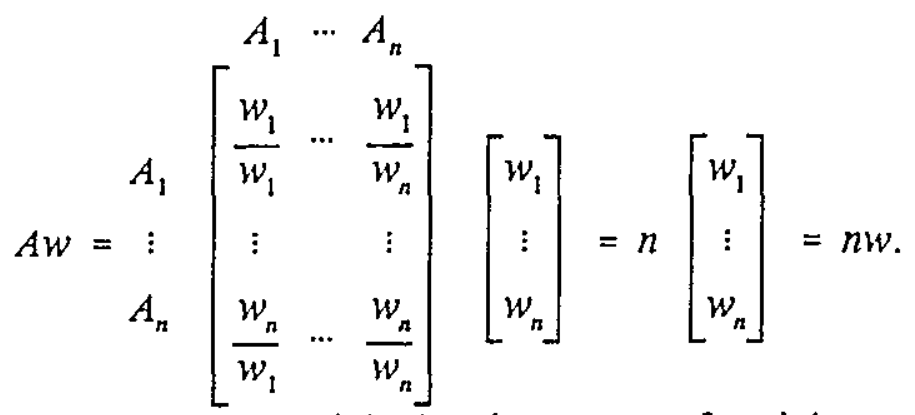

where $\mathrm{A}$ has been multiplied on the right by the vector of weights $w$. The result of this multiplication is $n w$. Thus, to recover the scale from the matrix of ratios, it is necessary to solve the problem $\mathrm{A} w=n w$ or $(\mathrm{A}-n \mathbf{I}) w=0$. This is a system of homogeneous linear equations. It has a nontrivial solution if and only if the determinant of A-nI vanishes, that is, $n$ is an eigenvalue of $\mathbf{A}$. Now $\mathbf{A}$ has unit rank since every row is a constant multiple of the first row. Thus all its eigenvalues except one are zero. The sum of the eigenvalues of a matrix is equal to its trace, the sum of its diagonal elements, and in this case the trace of $\mathbf{A}$ is equal to $n$. Thus $n$ is an eigenvalue of $\mathrm{A}$, and one has a nontrivial solution. The solution consists of positive entries and is unique to within a multiplicative constant.

To make $w$ unique, its entries can be normalized by dividing by their sum. Thus, given the comparison matrix, the scale can be recovered. In this case the solution is any column of A normalized. Notice that in A the reciprocal property $a_{j i}=1 / a_{i j}$ holds; thus, also $a_{i i}=1$. Another property of $\mathbf{A}$ is that it is consistent: its entries satisfy the condition $a_{j k}=a_{i k} / a_{i j}$. Thus the entire matrix can be constructed from a set of $n$ elements which form a chain across the rows and columns.

In the general case, assume one is given $n$ stones, $A_{l}, \ldots, A_{n}$, but that this time the weights $w_{l}, \ldots, w_{n}$ are unknown. The precise value of $w_{i} / w_{j}$ cannot be given, but instead only an estimate of it in the form of judgment. For the moment, consider an estimate of these values by an expert who is assumed to make small perturbations of the coefficients. This implies small perturbations of the eigenvalues. The problem now becomes $A^{\prime} w^{\prime}=\lambda_{\max } w^{\prime}$ where $\lambda_{\max }$ is the largest eigenvalue of $\mathrm{A}^{\prime}$. To simplify the notation we shall continue to write $\mathrm{A} w=\lambda_{\max } w$, where $\mathbf{A}$ is the matrix of pairwise comparisons. The problem now is: "How good is the estimate of $w$ ?" Notice that if $w$ is obtained by solving this problem, the matrix whose entries are $w_{i} / w_{j}$ is a consistent matrix. It is a consistent estimate of the matrix A. A itself need not be consistent. In fact, the entries of $A$ need not even be transitive; i.e., $A_{1}$ may be preferred to $A_{2}$ and $A_{2}$ to $A_{3}$ but $A_{3}$ may be preferred to $A_{1}$. What we would like is a measure of the error due to inconsistency. It turns out that $\mathrm{A}$ is consistent if and only if $\lambda_{\max }=n$ and that we always have $\lambda_{\max } \geq n$.

Since small changes in $a_{i j}$ imply a small change in $\lambda_{\max }$ the deviation of the latter from $n$ is a deviation from consistency and can be represented by $\left(\lambda_{\max }-n\right) /(n-1)$, which is called the consistency index (C.I.). When the consistency index has been calculated, the result is compared with those of the same index of a randomly generated reciprocal matrix from the scale 1 to 9 , with reciprocal requirements forced. This index is called the random index (R.I.). The following gives the order of the matrix $n$, and the corresponding average R.I. as calculated from hundreds of thousands of randomly generated matrices: 


\begin{tabular}{lcccccccccc}
\hline$n$ & 1 & 2 & 3 & 4 & 5 & 6 & 7 & 8 & 9 & 10 \\
\hline Random Consistency Index (R.I.) & 0 & 0 & .52 & .89 & 1.11 & 1.25 & 1.35 & 1.40 & 1.45 & 1.49 \\
\hline
\end{tabular}

The ratio of C.I. to the average R.I. for the same order matrix is called the consistency ratio (C.R.) A consistency ratio of 0.10 or less is positive evidence for informed judgment.

The 'relations $a_{j i}=1 / a_{i j}$ and $a_{i i}=1$ are preserved in these matrices to improve consistency. The reason for this is that if stone $\# 1$ is estimated to be $k$ times heavier than stone $\# 2$, common sense requires that stone $\# 2$ be estimated to be $1 / k$ times the weight of the first. If the consistency ratio is significantly small, the estimates are accepted; otherwise, an attempt is made to improve consistency by obtaining additional information. What contributes to the consistency of a judgment are (1) the homogeneity of the elements in a group, that is, not comparing a grain of sand with a mountain; (2) the number of elements in the group - to improve consistency we agree with the psychological experiments, which we can justify mathematically, that an individual cannot compare simultaneously more than seven objects (plus or minus two) without becoming more and more inconsistent [17]; and (3) the knowledge of the analyst about the problem under study.

For quantifying judgments, a fundamental scale is used with values ranging from 1 to 9 as shown in Table 1. There are several reasons for choosing such a scale [25]:

(1) The qualitative distinctions are meaningful in practice and have an element of precision when the items being compared are of the same order of magnitude or close together with regard to the property used to make the comparison.

(2) The ability to make qualitative distinctions is well represented by five attributes: equal, moderate, strong, very strong, and extreme and interpolations between them when desired.

(3) The consistency limit of $7 \pm 2$ items in simultaneous comparisons suggests that if $7+2$ elements are compared and if they are all slightly different from one another, a nine point scale woüld be needed to distinguish among these differences.

We note that the numerical values used in the scale are absolute rather than ordinal numbers. If numbers larger than those appearing in the scale are needed, the hierarchy itself is used to cluster the elements, and to compare the clusters before comparing their elements. Thus we assume that the factors being compared fall within the same order of magnitude implied by the scale.

\section{An Example of Reciprocal Comparisons and Derived Measurement}

Light radiation and light reflection depend on the source plus other factors, such as scattering and interference, and on the nature of the background. If one uses a point source of light, that source would radiate or diffuse luminance uniformly in all directions according to the inverse square law. Indeed some objects would rediffuse the light falling on them, rather than reflect it, and something perhaps closer to an inverse fourth power could apply. However, if the source is a directed beam confined to a narrow cone over the desired range, the objects would reflect the light according to the inverse square of the distance. An intervening object reflects the beam at an angle equal to the angle of incidence. 
Table 1

\begin{tabular}{|c|c|c|}
\hline $\begin{array}{l}\text { Intensity of } \\
\text { Importance }\end{array}$ & Definition & Explanation \\
\hline 1 & Equal Importance & Two activities contribute equally to the objective \\
\hline 3 & Moderate importance & $\begin{array}{l}\text { Experience and judgment slightly favor one } \\
\text { activity over another }\end{array}$ \\
\hline 5 & Strong importance & $\begin{array}{l}\text { Experience and judgment strongly favor one } \\
\text { activity over another }\end{array}$ \\
\hline 7 & $\begin{array}{l}\text { Very strong or demonstrated } \\
\text { importance }\end{array}$ & $\begin{array}{l}\text { An activity is favored very strongly over } \\
\text { another; its dominance demonstrated in practice }\end{array}$ \\
\hline 9 & Extreme importance & $\begin{array}{l}\text { The evidence favoring one activity over another } \\
\text { is of the highest possible order of affirmation }\end{array}$ \\
\hline $2,4,6,8$ & Intermediate Importance & $\begin{array}{l}\text { When one hesitates between the corresponding } \\
\text { upper and lower values. }\end{array}$ \\
\hline $\begin{array}{l}\text { Reciprocals } \\
\text { of above }\end{array}$ & $\begin{array}{l}\text { If activity } i \text { hus one of the above } \\
\text { nonzero numbers assigned to it when } \\
\text { compared with activity } j \text {, then } j \text { has the } \\
\text { reciprocal value when compared with } i\end{array}$ & A reasonable assumption \\
\hline Rationals & Ratios arising from the scale & $\begin{array}{l}\text { If consistency were to be forced by obtaining } n \\
\text { numerical values to span the matrix }\end{array}$ \\
\hline
\end{tabular}

Four identical chairs were placed on a line from a light source at the distances of 9,15 , 21 , and 28 yards. The purpose was to see if one could stand by the light and look at the chairs and compare their relative brightness in pairs, fill in the judgment matrix and obtain a relationship between the chairs and their distance from the light source. This experiment was repeated a number of times each involving a judgment matrix for which the relative brightness of the chairs was derived.

Let $C_{1}, C_{2}, C_{3}, C_{4}$ stand for the chairs, arranged in a straight line, leading away from the light. Judgments are obtained from an individual who stands by the light source and is asked, for example, "How strongly brighter is chair $\mathrm{C}_{2}$ than chair $\mathrm{C}_{3}$ ?" $\mathrm{He}$ would then give one of the numbers for the comparisons described in the table and each judgment is entered in the appropriate position in the matrix. By convention, the comparison of strength is always of an activity appearing in the column on the left against an activity appearing in the row on top. Two sets of people provided the judgments for each of the following two matrices:

\begin{tabular}{c|cccc} 
Brightness & $C_{1}$ & $C_{2}$ & $C_{3}$ & $C_{4}$ \\
\hline$C_{l}$ & 1 & 5 & 6 & 7 \\
$C_{2}$ & $1 / 5$ & 1 & 4 & 6 \\
$C_{3}$ & $1 / 6$ & $1 / 4$ & 1 & 4 \\
$C_{4}$ & $1 / 7$ & $1 / 6$ & $1 / 4$ & 1
\end{tabular}

\begin{tabular}{c|cccc} 
Brightness & $C_{1}$ & $C_{2}$ & $C_{3}$ & $C_{4}$ \\
\hline$C_{1}$ & 1 & 4 & 6 & 7 \\
$C_{2}$ & $1 / 4$ & 1 & 3 & 4 \\
$C_{3}$ & $1 / 6$ & $1 / 3$ & 1 & 2 \\
$C_{4}$ & $1 / 7$ & $1 / 4$ & $1 / 2$ & 1
\end{tabular}

The relative brightness of the chairs expressed by the principal eigenvectors (approximately) satisfies the inverse square law of optics. First we have: 
Relative brightness eigenvector

(1st Trial)

0.61

0.24

0.10

0.05
Relative brightness eigenvector

(2nd Trial)

0.62

0.22

0.10

0.06

The consistency of these judgments are measured by the principal eigenvalue of the reciprocal matrix as follows:
$\lambda_{\max }=4.1$
$\lambda_{\max }=4.39$
C.I. $=0.03$
C.I. $=0.13$
C.R. $=0.03$
C.R. $=0.14$

The relative brightness is obtained by computing the inverse square values. We have:

\begin{tabular}{cccccc}
\hline Distance & $\begin{array}{c}\text { Normalized } \\
\text { distance }\end{array}$ & $\begin{array}{c}\text { Square of } \\
\text { normalized } \\
\text { distance }\end{array}$ & $\begin{array}{c}\text { Reciprocal } \\
\text { of previous } \\
\text { column }\end{array}$ & $\begin{array}{c}\text { Normalized } \\
\text { reciprocal }\end{array}$ & $\begin{array}{c}\text { Rounding } \\
\text { off }\end{array}$ \\
\hline 9 & 0.123 & 0.015129 & 66.098 & 0.6079 & 0.61 \\
15 & 0.205 & 0.042025 & 23.79 & 0.2188 & 0.22 \\
21 & 0.288 & 0.082944 & 12.05 & 0.1108 & 0.11 \\
28 & 0.384 & 0.147456 & 6.78 & 0.0623 & 0.06 \\
\hline
\end{tabular}

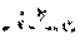

First and second trial eigenvectors should be compared with the last column of this table. It is interesting to note that the judgments have captured a natural law.

Note the sensitivity of the results as the object is very close to the source, for then it absorbs most of the value of the relative index and a small error in its distance from the source yields great error in its value.

The effectiveness of the fundamental semantic scale in making paired comparisons has been demonstrated in a variety of applications. One of these applications involves comparison of areas of five figures, done a large number of times in the presence of an audience due to its simplicity.

Figure 1 gives five areas to which the paired comparison process and the scale can be applied to test the validity of the procedure. One may approximate the outcome by adding the rows of the matrix and dividing by the total.

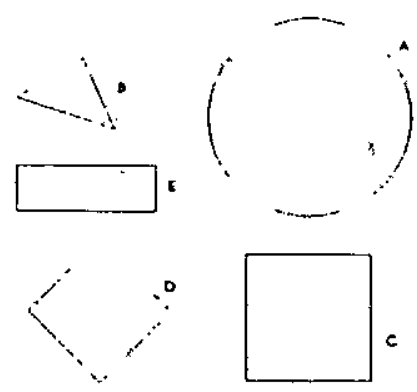

Figure 1 
The actual relative values of these areas are $A=0.47, B=0.05, C=0.24, D=0.14$, and $E=0.09$ with which the answer may be compared. By comparing more than two alternatives in a decision problem, one is able to obtain better values of the derived scale because of redundancy in the comparisons which helps improve the accuracy of the judgments.

\section{Continuous Comparisons -- Mental Activity and Ratio Scales}

In the first part of the paper we spoke of discrete comparisons and dealt with them as they occur at the conscious level. We now discuss continuous comparisons which occur at the unconscious level and most profoundly in the neurons of the brain. There are direct analogies to the case of discrete comparisons. All sensation, perception, feeling, behavior and thought are carried out by the firing of neurons. Neurons occur in a variety and use different neurotransmitters and neuropeptides in their type of communication. Through the firing process a neuron shares with other neurons with which it is linked in the representation of data and in the intensity with which these data are recorded. There are several consequences of neural firings the most important of which are the formation of pictures and sounds, detection of movement, the creation of feelings and ideas and the control of physical, behavioral, communication and thought processes to produce actions and reactions and to use memory and feedback to modify and change the control. All these occur according to carefully prescribed magnitudes mostly in proportion to the stimuli received. When precision is needed, which is often, the brain works with ratio scales to maintain correspondence between what is experienced and what is internalized.

What can we say about the relationship between the firing of neurons and the creation of patterns of recognizable information? How are mathematics and particularly numbers derived from the activity of neurons? This is a vast field for investigation for which we give a small start in this paper. As a byproduct of the basic idea of relative comparisons, we attempt to show how geometry occurs in sighted person's consciousness. We derive a general representation of the firing of neurons.

A neuron consists of three parts, the axone, the cell body and the dendrites [8]. The cell body processes information, whereas the dendrites receive signals and the axone sends signals. The synaptic cleft is a microscopic gap of about 200 nanometers width between the nerve endings of one neuron and the cell-body and dendrites of the next. The diode-like synapse conducts current in one direction only from the presynaptic to the postsynaptic neuron.

The firing of a neuron is influenced by its threshold of excitability, its local chemical environment, and the sum of excitatory and inhibitory impulses it receives within a brief period of time. The impulse delivered by a nerve at the various synapses has a constant amount of excitation or of inhibition, which are increased in amount by increasing the number of impulses sent in a short period. The impulse delivered to a neuron has to be raised to excite it to fire again and again. The excitatory effect of an impulse lasts 5 milliseconds. The intensity of the current supplied to a neuron and the neuron's rate of firing of impulses is linear in some neurons and has an S-shaped curve in others. Some places neurons in the central nervous system are kept receiving excitatory impulses so they would be ready to fire on the arrival of a few more impulses.

Some neurons spread their synapses widely and some channel them to a few pathways. When a central or linearly arranged group of neurons is excited, the surrounding neurons are simultaneously inhibited, and to emphasize contrast the stronger the excitation, the stronger is the surrounding inhibition. In addition, this type of excitation and inhibition can travel down 
a part of the body for example (lateral inhibition).

At the synapse excitation and inhibition are electrical occurrences similar to those in the nerve fiber. An impuise arrives at a synapse, causes the bursting of vesicles containing constant amounts of neurotransmitter chemical (made in the cell body and transported to the cleft) which travels across the cleft and unites with the protein of the postsynaptic membrane. The membrane then becomes permeable to some hydrated ions and not to others; according to which ions pass, and depolarization or excitation, or hyperpolarization or inhibition occur. Excitation and inhibition are determined by the size and shape of the pores of the receptor site. Smaller pores cause inhibition by allowing potassium and chloride ions to pass. Larger pores cause excitation by allowing sodium and chloride ions to pass. Once the transmitter has reacted with the postsynaptic membrane, it is inactivated and/or taken up again into the presynaptic nerve ending. In a chain of nerve fibers in the central nervous system, different transmitters are used. Even a simple reflex, with only one synapse, is not the same on every occasion. Repeated stimulation will decrease and stop the response.

According to Gregory [8], elaborate sets of wiring produce specificity of orientation and of direction of movement and other special properties. What is common to all regions is the local naturel of the wiring. By the time several synapses have been traversed the influence of the impact has spread vertically to all cell layers. Several other sets of fibers carry modified messages out of the area. The information carried into the cortex by a single fiber can make itself felt through the entire thickness. A block of cortex about one or two millimeters in size is what is needed to take care of a region of the visual world equivalent to the size of an aggregate field.

The reciprocal relation in neural firing arises in the comparison of neurotransmittergenerated charges in increments of time [27]. This comparison is represented by the reciprocal kernel mapping $K(s, t)$ where $s$ and $t$ denote time used for indexing as $i$ and $j$ are used in the discrete case. We select an interval of time $[a, b]$ and our convention will be to choose $a=0$. Let $0=t_{0}<t_{1}<\ldots<t_{n-1}<t_{n}=b$ be a partition of the interval $[0, b], I_{k} \equiv t_{k}-t_{k-1}, k=1,2, \ldots, n$. Let $w(t), t \in[0, b]$ be a single firing (voltage discharge) of a neuron in spontaneous activity. In simple term's, if $G(t), t \in[0, b]$ is the cumulative response of the neuron in spontaneous activity over time, given by $\mathrm{G}(\mathrm{t})=\int_{0}{ }^{t} w(u) d u$, then $\frac{d G(t)}{d t}=w(t)$, where $w(t) d t$ is the response during an infinitesimal period of time. Note that $G(t)$ is monotone strictly increasing and hence $w(t)>0$. L'et

$$
K\left(I_{i}, l_{j}\right) \equiv \frac{G\left(t_{i}\right)-G\left(t_{i-1}\right)}{G\left(t_{j}\right)-G\left(t_{j-1}\right)}
$$

be the relative comparison of the response of a neuron during a time interval of length $\Delta t_{i}$ with another time interval of length $\Delta \mathrm{t}_{\mathrm{j}}$. Cross multiplication and summation over $\mathrm{j}$ yields:

$$
\frac{1}{n} \sum_{j=1}^{n} K\left(I_{i}, l_{j}\right)\left[G\left(t_{j}\right)-G\left(t_{j-1}\right)\right]=G\left(t_{i}\right)-G\left(t_{i-1}\right), i=1,2, \ldots, n
$$

If $\mathrm{G}(\mathrm{t})$ is of class $\mathrm{C}^{\mathrm{l}}[0, \mathrm{~b}]$ of once differentiable functions, then as $\Delta \mathrm{t}_{\mathrm{k}} \rightarrow 0$ for all $\mathrm{k}$, $\mathrm{K}\left(\mathrm{I}_{\mathrm{i}}, \mathrm{I}_{\mathrm{j}}\right) \rightarrow \underset{\mathrm{K}}{\mathrm{K}}(\mathrm{s}, \mathrm{t})=\frac{w(s)}{w(t)}, s, t \in[0, b]$. Also because the left hand side of $(1)$ is an average, we obtain as $\Delta \mathrm{t}_{\mathrm{k}} \rightarrow 0$ for all $\mathrm{k}$, and as $\mathrm{n} \rightarrow \infty$ : 


$$
\frac{1}{b} \int_{0}^{b} K(s, t) w(t) d t=w(s)
$$

It is easy to show that $b$ is the principal eigenvalue (i.e., the largest in absolute value) of the consistent kernel $\mathrm{K}(\mathrm{s}, \mathrm{t})$ because all the other eigenvalues are zero, (the eigenfunctions being any function orthogonal to $\mathrm{w}(\mathrm{t})$ on $[0 ; \mathrm{b}]$.) In general, if $\mathrm{K}\left(\mathrm{I}_{\mathrm{i}}, \mathrm{I}_{\mathrm{j}}\right)$ is reciprocal but not consistent, the homogeneous equations takes the form given by:

$$
w(s)=\lambda_{0} \int_{0}^{b} K(s, t) w(t) d t \text { or in operator form }\left(I-\lambda_{0} K\right) w=0, w \in L_{2}[0, b]
$$

where $\mathrm{I}$ is the identity operator, and $\mathrm{K}$ is a compact integral operator defined on the space $\mathrm{L}_{2}[0, \mathrm{~b}]$ of Lebesgue square integrable functions:

$$
K f(s)=\int_{0}^{b} K(s, t) f(t) d t, f \in L_{2}[0, b]
$$

Because positive reciprocal kernels are non-factorable (the property that corresponds to irreducibility for non-negative matrices), there exists a unique positive simple eigenvalue $\lambda_{0}^{-1}$ whose modulus dominates the moduli of all other eigenvalues.

As in the discrete case, there is an eigenfunction $w(s)$, that is unique to within a multiplicative constant (thus belongs to a ratio scale), which corresponds to the simple maximum positive eigenvalue $\lambda_{0}^{-1}$. It is called the response function of the neuron in spontaneous activity. From $\mathrm{K}(\mathrm{s}, \mathrm{t})>0, \mathrm{~K}(0, \mathrm{t})=0$ for all $\mathrm{t} \neq 0$, it follows that $w(s)>0$, for $s \neq 0$, and $w(0)=0[29$, p.186]. If the reciprocal kernel $K(s, t) \geq 0$, on $0 \leq s, t \leq b$, is Lebesgue square integrable and continuously differentiable, and if $\lim _{\xi \rightarrow 0} K(\xi s, \xi i)$ exists, then:

$$
w(t)=r^{(x} e^{g(t)} / \int_{0}^{b} t^{\alpha} e^{g(t)} d t
$$

satisfies (2) for some choice of $\mathrm{g}(\mathrm{t})$. This solution of (2) assumes that the comparison process is continuous, but it is not meaningful to compare the response during an interval of length zero with the response during a non-zero interval no matter how small it is, for then the reciprocal comparison would be unbounded. From a theoretical standpoint, one can study the problem using Lebesgue integration and allowing only one zero.

Because linear combinations of the functions $\left\{\mathrm{t}^{\wedge} \mathrm{e}^{-\beta \mathrm{t}}, \alpha, \beta \geq 0\right\}$ :

$$
\sum_{i=1}^{n} \gamma_{i} t^{\alpha_{i}} e^{-\beta_{i}}, \gamma_{i} \in R_{\perp} \text {, for all } i
$$

are dense in the space of bounded continuous function $C[0, b]$ we can approximate $t^{\alpha} e^{g(t)}$ by linear combinations of $t^{\alpha} \mathrm{e}^{-\beta t}$ and hence we substitute $\mathrm{g}(\mathrm{t})=-\beta \mathrm{t}, \beta \geq 0$ in the eigenfunction $\mathrm{w}(\mathrm{t})$. In fact such linear combinations are dense in even more general spaces worthy of consideration in representing neural responses to stimuli [27]. The sensitivity of neural firing is not completely analogous to the density of the rational numbers in the real number system. The rationals are countable infinite, the number of neurons is finite but large. In speaking of density here we may think of coming sufficiently close (within some prescribed bound rather than arbitrarily close).

The firing of a neuron between waiting periods has been represented mathematically by the function:

$$
T(\alpha, \beta)=x^{\alpha}(t) e^{-\beta \operatorname{rr}(t)}=e^{\operatorname{\alpha log} x(t)-\alpha x(l)}, \quad x, \alpha, \beta>0
$$

for a single neuron, where $x$ is the intensity of a stimulus and $\alpha$ and $\beta$ are parameters having 
to do with the quantity of neurotransmitter input needed to build up to firing threshold and hence determines both the frequency and intensity of firings, and with the efficiency rate of using the amounts of available input respectively. The empirical work of Hodgkin and Huxley [9] show that this is in fact the expression that describes the firing of a neuron penetrated with an electric probe and subjected to a visual electric stimulus.

The analogous expression for the firing of a group of neurons is given by:

$$
T\left(\alpha_{1}, \ldots, \alpha_{n}, \beta_{i}, \ldots, \beta_{n}\right)=\sum_{i=1}^{n} \sum_{k=1}^{n} x^{\pi_{1}}\left(t-T_{k}\right) e^{-\beta_{r}\left(t-T_{k}\right)}
$$

where $\mathrm{T}_{\mathrm{k}}$ are time displacements of firings from absolute time $t$. This expression could provide the basis to account for the functions of the brain described above. In particular we have used these dense functions to show how pictures are formed [28].

We take the derivatives of $\mathrm{T}(\alpha, \beta)$, its gradient and normal to the gradient. Differentiation yields:

$\frac{\partial^{n} T}{\partial \alpha^{n}}=\log ^{n} \times T(\alpha, \beta)$ and in particular $\frac{1}{T} \frac{\partial T}{\partial \alpha}=\log x$ which says that the relative rate of change in response buildup varies with the logarithm of the magnitude of the stimulus. We also have: $\frac{\partial^{n} T}{\partial \beta^{n}}=(-1)^{n} x^{n} T(\alpha, \beta)$ and $\frac{1}{T} \frac{\partial T}{\partial \beta}=-x$ which says that the relative rate of change :of response decay declines with the magnitude of the stimulus.

Again $\frac{\partial^{l} T_{T}}{\partial \beta^{k} \partial \alpha^{n-k}}=(-1)^{k} x^{k} \log ^{n-k} X T(\alpha, \beta)=\left(-\frac{x}{\log x}\right)^{k} \log ^{n} X T(\alpha, \beta)$

Finally we have $\frac{1}{T} \frac{\partial T}{\partial x}=\frac{\alpha}{x}-\beta$ which says that the relative change in response to a stimulus of magnitude $x$ is a hyperbolic function of $x$.

The maximum increase in $\mathrm{T}$ is given by the gradient: $\nabla \mathrm{T}=\mathrm{T}(\alpha, \beta)(\log x,-x)$ and no increase is given by the normal to the gradient which lies in the tangent plane and is given by $\perp \Delta T=T(\alpha, \beta)\left(\frac{x}{\log x}, 1\right)$ which indicates stable response to a stimulus along the two parameters. More generally, if $T\left(\alpha_{1}, \ldots, \alpha_{n} ; \beta_{1}, \ldots, \beta_{n}\right)=\sum_{i=1}^{n} a_{l} x^{\alpha_{1}} e^{\beta_{n} x}$ then $\nabla T=T(\log x, \ldots, \log x,-x, \ldots,-x)$ and $\perp \nabla T=T(x / \log x, \ldots, x / \log x, 1, \ldots, 1)$.

In the above expression for the normal to the gradient there appears the expression $x / \log$ $x$, well known in number theory. According to the prime number theorem if $\pi(x)$ is the number of primes less than or equal to $x$ then $\lim _{x \rightarrow \infty} \frac{\pi(x)}{x / \log x}=1$. We conjecture this could be the cognitive connection between the firing of neurons and the origin of neurons. The nervous system must have an intrinsic way to estimate magnitudes to control the release of chemicals in proportionate amounts.

7. The Case for How the Mind Constructs Geometry [14,22]

There are sequences of sensations to which we are constantly exposed throughout life. The frequent recurrence of some sensations leads to a process of grouping them together to form concepts. These concepts are stored in memory and associated with similarly recognizable experiences. Identification of specific sensations is a discrete process that occurs in a succession 
and can be put into 1-1 correspondence with a set of consecutive integers. Sensations can also forcibly occur (be interpolated in memory) between two such discrete complex sensations. Interpolations between the integers can be associated with the rational numbers because they can be put into 1-1 correspondence with the integers. By considering all finite or infinite sequences of interpolations we are led to the topological equivalent of the real numbers and the one dimensional continuum. This continuum represents subjective time, which is the flow of information gathered from sensation. Subjective time flows in one direction and is irreversible. A later memory is associated with a greater number of sensation complexes than an earlier one. The information is gathered through many channels involving the senses and combinations of conscious and semiconscious streams. We note that the correspondence made with the integers does not depend on where we choose an origin because the correspondence may begin with any new experience of sensations. Thus our frame of reference, which is subjective time, is translation invariant. The one to one correspondence can now be made with any set of real numbers ordered as the original set without affecting the sequence of sensations itself. This is invariance under a continuous, one to one, order preserving transformation. We now have both translation and change of scale invariance.

The result is an isomorphic mapping from the real world to memory. It is not an identity mapping because subjective time is irreversible and cannot repeat. However, the sensations can be repeated and put in one-to-one correspondence with intervals of subjective time. Thus, the world of sensations is homomorphic to the one-dimensional irreversible continuum. So far we may conclude that the world of experience is essentially two dimensional, the dimension of sensations and the dimension of memories of sensations.

But usually there is a choice about acquiring or avoiding sensation and this experience can be analyzed as a sequence of interaction and avoidance of sensation. This can in turn be put in one to one correspondence with subjective time. We now have three dimensions to consider: subjective time, and the two dimensions of interaction and avoidance - all homomorphic to the real number continuum. The result is a topological space with a transformation group. The transformations in fact are an algebraic field. This field admits the usual topology, is locally compact, connected and satisfies the second countability axiom. As a result the topological algebraic field is homomorphic to one of three fields: the real numbers, the complex numbers, or the real quaternions. The translation group has the dimension of the full group. The foregoing argument can be generalized to the case where simultaneous sensation complexes are registered in memory as with the eye viewing different happenings at one time. This shows that our space-time is uniquely 4-dimensional, one dimension of time and three dimensions of space and is a metrizable space. In such a space it is possible to derive ratio scale eigenvectors and eigenfunctions. A useful way for relating two eigenvector ratio scales $x=\left(x_{1}\right.$, $\left.\ldots, x_{n}\right)$ and $y=\left(y_{1}, \ldots, y_{n}\right)$ is through Hilbert's order preserving metric defined by the ratios:

$$
D(x, y)=\log \left(\max _{i}\left(x_{i}, y_{i}\right) / \min _{i}\left(x_{i}, y_{i}\right)\right) \text {. }
$$

Clearly, the smaller the value of $\mathrm{D}(x, y)$, the closer together are $x$ and $y$.

An important concern in relating people as observers to forms in the environment is with the constancy or slow change in recognition of form, that is, of the characteristics of that form which remain invariant to proportion (of light in the case of vision) or movement of either the object itself or the eyes or head or body of the receiver. Constancy or invariance is essential to the duration of a form in the mind. Incremental change in form makes it possible to connect in small steps different states of that form. The states of a form may be regarded as 
decompositions of that form at different instants of time. An impression must be constantly renewed to register it in memory from instant to instant. Hence the idea of transformation and invariance with respect to transformation for the sake of recognition, connectedness and continuity, basic concepts in geometry.

\section{Spatial Information and Spatial Knowledge}

In this section and in the final one we discuss the abstract congruence of structures derived from sense data no matter what organ of the senses is used. The visual system is, by design, well suited for the acquisition of information about the extension of spatial features and about their anrangement in space. Other perceptual systems do provide some information about space; but, in respect to the quantity, accuracy, dependability, distribution, and timeliness of the spatial information they can provide, they are vastly inferior to the visual system [6].

According to Rieser [21] three issues are involved in how vision is developed. Each issue has two points of view. walk;

1) People come to know the spatial structure of things encountered sequentially along a

a) Spatial knowledge results from the construction of a cognitive map for assembling spatial data and coordinating them like jigsaw puzzle pieces within a unifying framework [31]. Cognitive map theorists assume that the capacity to create knowledge of novel relations resides first in the ability to integrate sequentially viewed objects within a single mentally represented coordinate system; and then, in the ability to read the new relations from that representation. Unlike perceptual information, cognitive information is not obtained directly from the space in which the task is performed [6]. It is, instead, obtained from memory. The information supplied by memory is either a representation of perceptual information, or information established by inference (e.g., relationships among spatial features that were not observed on the same occasion). Demonstrations have indicated that by the end of the first month, infants will make directionally appropriate arm movements to a visually perceived target $[20,10]$. For blind pedestrians, memory representation is constructed with piecemeal information acquired on many different occasions. It is probably less accurate than the representation that is constructed with information gathered on one, or at most a very few occasions by sighted people. For instance, because visual observers can observe a relatively large space on one occasion, they can observe not only its features, but also the spatial relationship of those features. Blind observers must bring together spatial information gathered on different occasions and establish the spatial relationship of many features by inference. The memorial representations of blind pedestrians are also different from the memorial representations of sighted pedestrians in respect to the quantity of information they contain. The predominant, mental representation perspective is that visual experience is necessary in order for people to encode spatial structure. Researchers agree that many congenitally blind people seem to have little "spatial sense" although they mentally represent spatial layout of large spaces.

b) There are direct perceptions by which observers detect spatial structure, perceptions mediated by perceptual learning, not by mentally constructed maps. This view, proposed by James Gibson [7], emphasizes that knowledge of the spatial structure of places viewed in succession along a walk can be explained without attributing the creative work of spatially interrelating the different views to the construction of a cognitive map. The 
responsible process is perceptual learning.

2) What form does spatial knowledge take?

a) Such knowledge is necessarily visual, encoded in visual images or in nonimaginal visual terms; and

b) Such knowledge is analogous to the spatial information itself and not devoted to any particular sensory modality. Here we have two themes:

i) Descarte's view was that sequences of tactual impressions are compiled within a single, unifying spatial framework, a framework embodying the metric properties of Euclidean geometry. For Descartes, the Euclidean framework was amodal, able to register inputs from different senses in universal terms, and in this way it tended to unify the senses,

ii) Berkeley [2] denied Descarte's nativistic view of development, and claimed that the ability to learn the spatial structure of sequentially encountered things has an associationistic basis in previous experience.

3) How does spatial knowledge develop?

a) The capacity to know spatial structure is induced by visual experience and cannot develop without vision sometime during one's lifetime.

b) Visual experiences play a much weaker developmental role and the capacity can develop without vision.

We may assume that all information about the real world is acquired in relative terms through paired comparisons and paired comparisons give rise to inversion. We now briefly investigate the kind of knowledge derived through inversion.

\section{Brief Perspective on the History of Hyperbolic Geometry [30]}

Early in the 19th century, Gauss, Bolyai and Lobachevski deduced the logical consequences of replacing the parallel axiom of Euclidean geometry with the hyperbolic parallel axiom. The results were exercises in logic without a real model of that geometry. In the 1860's Eugenio Beltrami made hyperbolic geometry physically plausible by showing that there is local isometry between the two surfaces, the pseudosphere and the hyperbolic plane. Along with the sphere and the plane the pseudosphere has constant curvature and therefore its geodesics map into straight lines in the plane. Beltrami proved that a geodesic mapping of the pseudosphere into a Euclidean plane carries each point of the pseudosphere to a point of the unit disk. It is also known [5] that there is a 1-1 geodesic mapping of the hyperbolic plane onto the unit disk. This linkage of the local metric $d s^{2}$ of the pseudosphere and the hyperbolic plane through the unit disk shows that the two surfaces are locally isometric and hence that hyperbolic geometry has a 1-1 geodesic mapping into (but not onto) a surface in three dimensional Euclidean space. The geodesic connection through the unit disk as a representation of the hyperbolic plane and the various geodesic properties linked to the pseudosphere give the unit disk a special role as a model of the hyperbolic plane. It is called the Beltrami disk model. Based on a somewhat different approach, Felix Klein in 1871 again showed that the unit disk justifiably serves as a model of the hyperbolic plane. His points, lines, parallel lines, and diverging lines were the same as Beltrami's but he used a different idea of distance than a geodesic and of angle and of motion or transformation. 'Inversion with respect to the unit circle discussed in the next section came a little later as a model of hyperbolic geometry. 


\section{Inversion and Geometry $[1,3,16]$}

Inversion arises in all comparisons, not just with respect to distance. To see this, let $\mathscr{E}$ be an arbitrary set of a countable number of elements and let $\mathscr{E} \times \mathscr{E}$ be its cartesian product. For every $\mathrm{A} \in \mathscr{E}$ let $\mathrm{F}_{\mathrm{A}}: \mathrm{A} \times \mathscr{B} \rightarrow \mathscr{E} \times \mathrm{A}$ be a mapping that associates with each pair $(\mathrm{A}, \mathscr{E})$ $\in A \times \mathscr{E}$ the pair $(\mathscr{E}, \mathrm{A}) \in \mathscr{E} \times \mathrm{A}$. Let $\mathrm{K}: \mathscr{E} \times \mathscr{E} \rightarrow \mathrm{R}^{+}$be a mapping to the positive reals. If for any $B \in \mathscr{E}$ we have $K(A, B) K(B, A)=1$, where $(B, A)=F_{A}(A, B)$, then $F \equiv\left\{F_{A}: A \in\right.$ B) defines a reciprocal relation on $\mathscr{E} \times \mathscr{E}$. As we have seen, a reciprocal relation is an inversion.

Hyperbolic geometry is a characterization of optical reality. Geometry is studied in two ways. The first is structural in terms of metric properties giving rise to form, size, congruence and similarity, more in line with visual properties the eyes are able to perceive. The other equivalent approach is through groups of transformations.

The Euclidean plane $\mathrm{E}$ can be generated by mapping the interior of a circle with center $\mathrm{O}$ and radius $\mathrm{r}$ to its exterior by means of an involution or inversion $\gamma_{\mathrm{c}}$, i.e., a bijective mapping of the circle to itself such that $\gamma_{c}{ }^{2}=1$. This mapping makes each point $\mathrm{A}$ inside the circle correspond to a point $\mathrm{A}^{\prime}$ outside the circle such that $\mathrm{d}(\mathrm{O}, \mathrm{A}) \mathrm{d}\left(\mathrm{O}, \mathrm{A}^{\prime}\right)=1$. The inversive plane is then defined by adding $\{\infty\}$ to $E$ [16]. The points on the circle of inversion are fixed.

Poincare constructed a model to represent hyperbolic geometry in the Euclidean plane based on inversion with respect to a given circle of unit radius and center $O$. Here again the ..circle, and inversions with respect to it play an important role. If a curve intersects the circle : of inversion, its inverse will intersect it in the same point. The circle of inversion is fixed. Straight lines through the center of inversion are also fixed under inversion, although there is a redistribution of the points. Every circle orthogonal to the circle of inversion is fixed, for the power of the center of inversion with regard to such a circle is 1 . Inversion takes straight lines and circles to straight lines and circles. Circles may become straight lines and vice versa. If a moving point traces any curve continuously, its inverse will trace continuously a curve called the inverse of the first.

We give four important and typical theorems. Their geometric structure must have counterparts for other more usuál kinds of inversions.

Theorem: If $\mathrm{A}, \mathrm{A}^{\prime}$ and $\mathrm{B}, \mathrm{B}^{\prime}$ are any two pairs of inverse points which do not lie on the same diameter of the circle of inversion, then they lie on a circle and angles OAB and OBA are equal, respectively, to angles $O \mathrm{~B}^{\prime} \mathrm{A}$ and $\mathrm{OAB}$ '.

Theorem: Every straight line which does not pass through the center of inversion inverts into a circle passing through the center of inversion, and conversely.

Theorem: Circles which do not pass through the center of inversion invert into circles that pass through the circle of inversion.

When a transformation preserves angles it is said to be conformal.

Theorem: Inversion is a conformal transformation.

Inversions do not distinguish between Euclidean lines and Euclidean circles because their inversion is either a line or a circle. Thus, the term inversive lines is used to refer to Euclidean lines or Euclidean circles. A Euclidean line may be regarded as the 'limiting case' of circles of increasing radius. In this sense, it is not surprising that reflection in a line is the analog of inversion in a circle.

The representation of hyperbolic space by the inversive plane was mentioned casually by mathematicians in England, Germany, and the United States in the 19th century. Its first systematic development was in Germany by Heinrich Liebmann in his Nichteuklidische 
Geometrie [19].

The inversive plane has been used in two distinct, though related ways. Following Poincaré, the circles (and lines) orthogonal to one circle (or line) $\omega$ represent the lines of the hyperbolic plane, and the points inside the circle (or in the upper half-plane bounded by the line) represent the points of the hyperbolic plane. Second, following Liebmann, all the circles (and lines) in the inversive plane represent the planes of hyperbolic space. In this case, lines are represented by point-pairs, but points are not represented at all, unless it can be said that they are represented by elliptic bundles. The relation between these two representations is seen by taking Poincarés $\omega$ to be the Liebmann representation of one particular plane in hyperbolic space and considering the lines in this one plane, which is, of course, a hyperbolic plane. The representation generated by reciprocal comparisons belongs to the hyperbolic plane.

In the three dimensional case the circle may be conveniently regarded as a sphere: a celestial sphere that surrounds the observer and is so large that he may be regarded as being at its center even when he changes position. In this manner, the three-parameter family of planes in space is represented by the distinction made between great and small circles - that is, by the three-parameter family of circles in the inversive plane. Each pair of intersecting planes is represented by a pair of intersecting circles; the line of intersection is represented by two points, points at which the circles intersect - arising from the two ends of the line; and the two supplementary dihedral angles (formed by the two intersecting planes) are represented by the angles of intersecting, tangent, and on-intersecting - represent three kinds of plane-pair: intersecting, parallel, and ultraparallel. Thus, the space under consideration is hyperbolic, not Euclidean.

Inversion gives credibility to the idea that the geometric and more generally mathematical properties of the perceived world in which we live are closely linked to the fundamental operations on which our cognitive processes are based.

\section{Conclusion}

Granted that inversion which derives from comparisons gives rise to a mentally hyperbolic manifold, and to complete our effort to link the physical world to our thought processes, what guarantee do we have that our eyes and vision are structured and located in such a way as to represent without distortion space and time as a hyperbolic manifold that is compatible with the inversion required by our thought processes based on comparison?

In his seminal work Rudolf Luneberg [15] has investigated this question. His conclusion may be summarized as follows: He assumes that physical space is a Euclidean manifold of the "domain in which binocular vision is of practical importance." But then he goes on to show that if the physical world were Euclidean, our perception of the size of objects would always be in proportion to their distance which is not the case as evidenced for example by the moon and the sun. He calls this requirement of proportionality, psychometric coordination and proves that the geometric manifold required for psychometric coordination is unique. Since the geometry in a manifold can be derived from its metric (using small line elements), he shows by requiring isometry between the differentials of a Euclidean physical world and the visual manifold, that visual space as determined by binocular vision is non-Euclidean, and in fact hyperbolic.

One may question the idea that the eye sees the entire manifold at once and that if is does not, the resulting conclusions may be incorrect. To address this concern, Indow [11] showed that as a result of the constancy of the principle of reciprocal comparisons, the curvature of the optical space must be the same constant rather than a different one for each glance of the eye. 
Luneberg argued that there is no contradiction between the assumption of a locally Euclidean world and the conclusion that visual sensations form a non-Euclidean manifold, by showing that relativistic space of physical experience also leads to a hyperbolic metric. To do this he relied on the fact that the Minkowski space-time metric with differential element $d s^{2}=d x^{2}+d y^{2}+d z^{2}-c^{2} d t^{2}$ is hyperbolic.

The result is that what is known about physical space leads to a hyperbolic metric; it is sensed through binocular vision that is also hyperbolic and interpreted by a nervous system that applies comparisons to relate and understand sensations which lead to inversion and to hyperbolic geometry. One may infer from all this that there is a unifying principle of comparisons underlying and unifying all three, and is the same for the physical world, for our visual sensation and for our world of mental synthesis. In addition, because of the universality of inversion across all processes of sensing cognition, Descarte's dictum about the amodality of geometry becomes highly plausible with the exception that "Euclidean" should be replaced by "hyperbolic". Finally, because comparisons are essential for evaluating how we sense and feel the world, the idea of a metric and of its corresponding geometry is also essential for obtaining basic knowledge about the world. In this light, the classical idea that the physical world as - perceived by us is mathematical through and through appears reasonable and can now bé .extended to include our thinking minds in it.

\section{References}

1. Benedetti, H. and C. Petronio, 1992, "Lectures on Hyperbolic Geometry", Springer-Verlag, New York.

2: Berkeley, G., 1965, An Essay Toward a New Theory of Vision, In: D. Armstrong (ed), Berkeley's Philosophical Writings, pp. 275-352. MacMillan, NY.

3. Casson, 'A.J. and S.A. Bleiler, 1988, "Automorphisms of Surfaces after Nielsen and Thurston", London Mathematical Society Student Texts 9, Cambridge University Press.

4. Descartes, R., 1965, Discourse on Method, Optics, Geometry, and Meteorology, (P. Olscamp, Trans.), BobbsMerrill, New York.

5. Efimov, N.V., 1980, "Higher Geometry", Mir, Chapter 8, 474-528.

6. Foulke, E., 1984, "Spatial Information and Spatial Knowledge", In: Proceedings of The Louisville Space Conference, University of Louisville, KY, April 13-14, 1984.

7. Gibson, J.J., 1979, The Ecological Approach to Visual Perception, Houghton-Mifflin, Boston.

8. Gregory, R.L. (ed.), 1987, "The Oxford Companion to the Mind", Oxford University Press, Oxford, NY.

9. Hodgkin, A.L. and A.F. Huxley, 1952, "A Quantitative Description of Membrane Current and its Applications to Conduction and Excitation in Nerve", Journal of Physiology 117, 500-544.

10. Hofsten, C. von, 1982, "Eye-hand Coordination in the Newborn", Developmental Psychology 18, pp. 450-461.

11. Indow, Tarow, 1979, "Alleys in Visual Space", J. of Mathematical Psychology 19, pp. 221-258.

12. Landau, B., H. Gleitman, and E. Spelke, 1981, "Spatial Knowledge and Geometric Representation in a Child Blind from Birth", Science 213, pp. 1275-1278. 
13. Lebesgue, H., Leçons sur l'integration, 2nd ed., Gauthier-Villars, Paris, 1928.

14. Levine, M., I. Jankovic and M. Palij, 1982, "Principles of Spatial Problem"Solving.", J. of Experimental Psychology: General 111, pp. 157-175.

15. Luneburg, R.K., 1947, "Mathematical Analysis of Binocular Vision", Princeton University Press, Princeton, New Jersey.

16. Lyndon, R.C., 1987, "Groups and Geometry", London Mathematical Society Lecture Note Series 101, Cambridge University Press.

17. Miller, G.A., "The Magical Number Seven Plus or Minus Two: Some Limits on our Capacity of Processing Information", Psychological Review 63, pp. 81-97, 1956.

18. Monod, J., 1971, "Chance and Necessity; An Essay on the Natural Philosophy of Modern Biology", Knopf, New York.

19. The New Encyclopaedia Britannica, Non-Euclidean Geometry, 15th Ed., 1985, Encyclopaedia Britannica, Chicago.

20. Rader, N. and J.D. Stem, 1982, "Visually Elicited Reaching in Neonates", Child Development 53, pp. 10041007.

21. Rieser, J.J., "Mental Models and Direct Perception", In: Proceedings of The Louisville Space Conference, University of Louisville, KY, April 13-14, 1984.

22. Rothstein, J., 1962, "Wiggleworm Physics", Physics Today, Sept., pp. 28-38.

23. Saaty, T.L. and K.P. Kearns, 1985, Analytical Planning: The Organization of Systems, Pergammon Press, New York.

24. Saaty, T.L. and J.M. Alexander, 1989, Conflict Resolution: The Analytic Hierarchy Approach, Praeger, New York.

25. Saaty, T.L., 1990, Multicriteria Decision Making: The Analytic Hierarchy Process, RWS Fublications, Pittsburgh, PA.

26. Saaty, T.L. and L.G. Vargas, 1991, Prediction, Projection and Forecasting, Kluwer Academic, Boston.

27. Saaty, T.L. and L.G. Vargas, 1993, "A Model of Neural Impulse Firing and Synthesis", Mathematical Psychology 37/2, 200-218.

28. Saaty, T.L. and L.G. Vargas, 1994, "Representation of Visual Response to Neural Firing", Mathematical and Computer Modelling, to appear.

29. Shaposhnikova, T.O., R.S. Andersen and S.G. Mikhlin (Eds.), 1975, "Integral Equations", Leyden Noordhoff.

30. Shenitzer, A., May 1994, "How Hyperbolic Geometry Became Respectable", American Mathematical Monthly, 464-470.

31. Siegel, A.W. and S. White, 1975, "The Development of Spatial Representations of large-scale environments", In: H.W. Reese (Ed.), Advances in Child Development and Behavior, Vol 10, Academic Press, NY.

32. Sinclair, S., 1985, "How Animals See", Facts on File Publications, New York, p. 30.

33. Zeki, S., 1993, "A Vision of the Brain", Blackwell Scientific Publications, Oxford. 